\title{
Methicillin resistant Staphylococcus aureus contamination of phlebotomy tourniquets and faucets
}

\author{
T Abeywickrama ${ }^{1}, \mathrm{~K}$ Amarasinghe ${ }^{1}$, S Wijerathne ${ }^{1}$, C Dharmaratne ${ }^{1}$, D Fernando ${ }^{1}$, B C Senaratna ${ }^{2}$, \\ H A K M Gunasekera ${ }^{3}$
}

(Index words: MRSA, tourniquets, faucets, Sri Lanka)

\begin{abstract}
Introduction Methicillin resistant Staphylococcus aureus (MRSA) is transmitted through direct contact or fomites. The most important means of nosocomial spread is by hospital personnel. However, fomites are being increasingly recognized as sources of nosocomial infection.

Objectives Our aim was to describe the MRSA contamination rate of phlebotomy tourniquets and faucets in a tertiary care hospital and to compare the contamination of plastic tourniquets with that of fabric tourniquets.

Method A cross-sectional study was carried out in the general wards of a tertiary care hospital in the Colombo District. Two hundred tourniquets were collected and 100 faucets were swabbed and cultured on CHROMagar ${ }^{\mathrm{TM}}$ MRSA medium (CHROMagar Microbiology). Contamination rates of 50 plastic tourniquets and 50 fabric tourniquets were compared.

Results MRSA grew in $26 \%$ of tourniquets. Majority were plastic tubes. MRSA contamination of tourniquets did not significantly differ by ward $(p>0.4)$. MRSA was found on $26 \%$ of faucets. Contamination rate was highest in the common wards for dermatology, dental, rheumatology, and neurology (55.6\%), followed by gynaecology (45.2\%), cardiology $(33.3 \%)$, surgery $(18.8 \%)$, psychiatry $(11.1 \%)$, and medicine (5.6\%). There was a significant difference in rates of contamination of faucets in the different wards $(p<0.01)$. There was no significant difference in the colony count per surface area of the two types of tourniquets after a single use.

Conclusions MRSA contamination rates of tourniquets and faucets were high. Single-use new plastic tourniquets were much less contaminated with MRSA than reused tourniquets.
\end{abstract}

Ceylon Medical Journal 2018; 63: 5-10

DOI: http://doi.org/10.4038/cmj.v63i1.8627

\section{Introduction}

Methicillin-resistant Staphylococcus aureus (MRSA) is a leading cause of nosocomial infections [1]. The most important means of nosocomial spread is through carriage of S.aureus on the hands, scalp and anterior nares of healthcare workers [1]. However, fomites are being increasingly recognized as sources of nosocomial infections [2-4]. Disconcertingly high rates (81\%) of MRSA in clinical specimens with S.aureus have been reported in Sri Lanka [5].

The degree of MRSA contamination of inanimate objects is dependent on the colonization and infection rates of patients and healthcare workers in that unit [2]. It also depends on the cleaning and infection control measures of the institution [6]. In other countries, phlebotomy tourniquets and faucets have been identified as important reservoirs of MRSA [2-4,6,7]. Although causality between contaminated tourniquets and increased MRSA infection has not been established, the potential for cross-contamination cannot be ignored. This has been demonstrated in some studies [2,8,9]. It has been shown that health care staff contaminate their gloves by handling or touching sites near patients colonized with MRSA and that tourniquets are contaminated mainly via healthcare workers rather than patients [2,9]. Environmental cleaning and replacement of shared devices have been effective in controlling MRSA outbreaks which occur despite standard infection control measures [10,11].

Type, duration of use and disinfection of tourniquets reported in these studies are different from those in Sri Lanka. One main difference is that most government hospitals in Sri Lanka use plastic tubing from infusion sets as tourniquets. Therefore, we conducted this study to describe the MRSA contamination rate of tourniquets and faucets in a local tertiary care hospital. We also compared the degree of contamination of plastic tubing

${ }^{1}$ Faculty of Medical Sciences, University of Sri Jayewardenepura, ${ }^{2}$ Department of Community Medicine, Faculty of Medical Sciences, University of Sri Jayewardenepura, Sri Lanka, ${ }^{3}$ Department of Microbiology, Faculty of Medical Sciences, University of Sri Jayewardenepura, Sri Lanka.

Correspondence: HAKMG, e-mail: <kamani.lk@gmail.com>. Received 04 December 2017 and revised version accepted 05 January 2018.

This is an open-access article distributed under the terms of the Creative Commons Attribution License, which permits unrestricted use, distribution, and reproduction in any medium, provided the original author and source are credited. 
with that of commercially available fabric tourniquets to verify reports that smooth surfaces are less likely to be contaminated with bacteria than rough surfaces [12,13]. Standard precautions practised during phlebotomy in the chosen hospital, were also assessed.

\section{Methods}

This descriptive cross-sectional study was carried out in all wards except the intensive care and special care units of a tertiary care hospital in the Colombo District, Sri Lanka. We conducted this in four phases (see below) from September to December, 2014.

\section{Phase A - MRSA contamination rate of tourniquets}

We collected all tourniquets which were being used in the wards at the start of the study. As the total number of tourniquets collected from all wards during the first round ( $n=77$ ) was less than the calculated sample size of 200, more tourniquets were collected on two other occasions, one month apart (total $n=206$ ). The tourniquets were transported to the laboratory, in sterile bags on the same day.

Two of the wards used commercially purchased tourniquets. As these could not be removed, we swabbed the surfaces of these tourniquets with a moist sterile swab each time tourniquets were collected. A representative number of tourniquets were collected at random from wards that had single-patient tourniquets.

Using aseptic techniques these tourniquets were incubated overnight at $37^{\circ} \mathrm{C}$ in brain-heart infusion broth, $200 \mu \mathrm{l}$ of broth was sub-cultured on to CHROMagar ${ }^{\mathrm{TM}}$ MRSA medium (CHROMagar Microbiology, Paris, France; “CHROM”) and processed according to manufacturer's instructions. Two of the investigators independently examined the plates and those with pink colonies that had a colony morphology characteristic of staphylococci were reported as MRSA positive. Gram staining and coagulase tests were done for confirmation. All other colony types and absence of growth were recorded as MRSA negative.

\section{Phase B - MRSA contamination rate of faucets}

A representative number of faucets were randomly selected from different locations in 29 wards and a total of 100 faucets were swabbed. Faucets was grouped based on their location, namely: a. faucets in wards used mainly by health care workers b. faucets in wards used mainly by patients, c. faucets in the pantry, d. faucets in toilets used by health care workers, e) faucets in toilets used by patients, f) faucets in washing areas used for cleaning instruments (Supplementary table). A moist sterile swab rubbed over the entire surface of the faucet was incubated overnight in brain-heart infusion broth and sub-cultured on to CHROM as described above.
Phase C-Effect of tourniquet material on contamination rate

One or two patients were randomly selected from each ward during routine phlebotomy. Around each patient's arm one commercially available fabric tourniquets and one plastic tubing (both sterilized prior to use) were tied close together, without contact with each other ( $n=50$ pairs). We alternated the positions of tourniquets to avoid the site of application affecting the results. Following phlebotomy, the tourniquets were placed in separate sterile bags by the health care worker.

Maintaining aseptic precautions, two inch pieces from both ends and the middle part of each tourniquet were cut using sterile scissors and inoculated on to blood agar. Both sides of the cut pieces from the commercially available fabric tourniquets were pressed once on agar. Pieces from the plastic tubing were cut lengthwise and the whole outer surface was pressed once on agar. The inoculum was spread over the agar with a loop. After overnight incubation at $37^{\circ} \mathrm{C}$, the total number of colonies was recorded and suspect staphylococcal colonies were sub-cultured on to CHROM.

Phase D-Observation of standard precautions adopted during phlebotomy

We observed the routine phlebotomy procedures in all wards for a period of three weeks. Health-care workers were unaware of being observed; however, we allowed a grace period of three days between introduction of data collectors to the ward and recording of practices, to allow the health-care workers to be familiar with the presence of data collectors. Using an observational checklist, we collected data on four separate occasions in each ward. In two of the obstetrics and gynaecology wards, two of the ear nose and throat (ENT)/ophthalmology wards and one psychiatry ward, phlebotomy was performed very early in the morning and the presence of data collectors in these wards at these times would have made their purpose obvious to the health-care workers. Therefore, we excluded these five wards from this phase of the study.

\section{Statistical analysis}

Data were entered, cleaned, and analysed with SPSS version 21.0 software (IBM Corporation). Descriptive data are reported as frequencies and percentages. Factors associated with MRSA contamination were described using cross tabulations (with Fisher's exact test) and $p<0.05$ was considered to be statistically significant.

Ethics approval was obtained from the Ethics Review Committee of the Faculty of Medical Sciences, University of Sri Jayewardenepura.

\section{Results}

Twenty nine out of 32 wards (90.6\%) consisting of 
surgery $(\mathrm{n}=8)$, obstetrics and gynaecology $(n=8)$, medicine $(n=6)$, ENT/ophthalmology $(n=2)$, paediatrics $(n=1)$, cardiology $(n=1)$, psychiatry $(n=1)$ and two common wards with patients from dermatology, rheumatology, neurology and dentistry, were included. Two surgical wards that did not consent and one gynaecology ward that did not have tourniquets were excluded.

\section{Phase A - MRSA contamination rates of tourniquets}

Majority of the wards (27 of 29) used plastic tubing. Two wards used one plastic tubing per patient (singlepatient tourniquets; $n=14$ ) whereas the others $(n=192)$ reused a plastic tubing on multiple patients for periods varying from 12 hours to three days. Two wards had commercially purchased tourniquets; one of these wards used plastic tubing in addition to a commercially purchased plastic tourniquet which had been used for two months. The other had only a commercially available fabric tourniquets which had been used for 26 months. None of the tourniquets were visibly blood stained on collection.

Overall, 53 of 206 (25.7\%) tourniquets grew MRSA. The two commercially purchased tourniquets were swabbed on four occasions and the commercially purchased plastic tourniquet was found to be positive for
MRSA on two occasions. The commercially purchased plastic tourniquet had a higher contamination rate than the commercially available fabric tourniquets $(p<0.05$; Table 1). The contamination rate of the plastic tubing (not the commercially purchased ones) was $25.8 \%$ (51 of 198) and $28.6 \%$ of single-patient tourniquets were contaminated with MRSA. Neither the frequency of use (single-patient versus repetitive use) nor the specialty of the ward significantly affected contamination rates $(p=0.76$ and $p=0.13$ respectively; Table 1 ).

\section{Phase B - MRSA contamination rates of faucets}

MRSA was isolated from 26 of the 100 faucets which were randomly sampled. Contamination rate was significantly associated with the type of ward $(p<0.01)$. More than half the contaminated faucets were from the the gynaecology wards (Table 2). The highest contamination rate was in the two common wards which consisted of the specialities of dermatology, dental, rheumatology and neurology (55.6\%). The second highest rate was in the obstetrics and gynaecology ward (45.2\%), followed by cardiology (33.3\%), surgery (18.8\%), psychiatry (11.1\%) and medicine (5.6\%). Contamination rate did not significantly differ by location within the ward $(p=0.44)$ or user (health-care workers versus patients $p=0.11)$.

Table 1. Factors associated with MRSA positive tourniquets

\begin{tabular}{|c|c|c|c|}
\hline & $\begin{array}{r}\text { MRSA Contaminated }(n=53) \\
N(\%)\end{array}$ & $\begin{array}{r}\text { MRSA not contaminated }(n=153) \\
N(\%)\end{array}$ & $\begin{array}{r}\text { Significance } \\
(p \text { value })\end{array}$ \\
\hline \multicolumn{4}{|l|}{ Type of tourniquet } \\
\hline Commercial & $2(25.0)$ & $6(75.0)$ & 1.00 \\
\hline Non-commercial & $51(25.8)$ & $147(74.2)$ & \\
\hline Commercial fabric & $0(0.0)$ & $6(100)$ & 0.03 \\
\hline Commercial plastic & $2(100)$ & $0(0.0)$ & \\
\hline Plastic IV tubes & $51(25.76)$ & $147(74.24)$ & \\
\hline \multicolumn{4}{|l|}{ Type of ward } \\
\hline Surgery & $17(26.6)$ & $47(73.4)$ & 0.13 \\
\hline Medicine & $9(18.4)$ & $40(81.6)$ & \\
\hline Obstetrics/Gynaecology & $12(22.6)$ & $41(77.4)$ & \\
\hline Paediatrics & $6(50)$ & $6(50.0)$ & \\
\hline Cardiology & $5(55.6)$ & $4(44.4)$ & \\
\hline Psychiatry & $2(40.0)$ & $3(60.0)$ & \\
\hline ENT/Ophthalmology & $1(20.0)$ & $4(80.0)$ & \\
\hline Others $^{\mathrm{a}}$ & $1(11.1)$ & $8(88.9)$ & \\
\hline \multicolumn{4}{|l|}{ Type of use } \\
\hline Single-patient & $4(28.6)$ & $10(71.4)$ & 0.76 \\
\hline Re-used & $49(25.5)$ & $143(74.5)$ & \\
\hline
\end{tabular}

a (Dermatology, dental, rheumatology, and neurology)

*Fisher's Exact test 
Table 2. Factors associated with the MRSA positive faucets

\begin{tabular}{|c|c|c|c|}
\hline & $\begin{array}{r}\text { MRSA Contaminated } \\
(n=53) \\
N(\%)\end{array}$ & $\begin{array}{r}\text { MRSA not contaminated } \\
(n=153) \\
N(\%)\end{array}$ & $\begin{array}{r}\text { Significance } \\
(p \text { value })\end{array}$ \\
\hline \multicolumn{4}{|l|}{ Type of ward } \\
\hline Surgery & $3(18.8)$ & $13(81.3)$ & $0.003 *$ \\
\hline Medicine & $1(5.6)$ & $17(94.4)$ & \\
\hline Obstetrics/Gynaecology & $14(45.2)$ & $17(54.8)$ & \\
\hline Cardiology & $2(33.3)$ & $4(66.7)$ & \\
\hline Psychiatry & $1(12.5)$ & $8(87.5)$ & \\
\hline ENT/Ophthalmology & $0(0)$ & $10(100)$ & \\
\hline Paediatrics & $0(0)$ & $1(100)$ & \\
\hline Others $^{\mathrm{a}}$ & $5(55.6)$ & $4(44.4)$ & \\
\hline Location of faucet & & & 0.44 \\
\hline Ward & $3(15.0)$ & $17(85.0)$ & \\
\hline Pantry & $1(12.5)$ & $7(87.5)$ & \\
\hline Toilet/bathroom & $21(31.3)$ & $46(68.7)$ & \\
\hline Washing area & $1(20.0)$ & $4(80.0)$ & \\
\hline Type of user & & & 0.11 \\
\hline Healthcare worker & $7(17.1)$ & 34 (82.9) & \\
\hline Patient & $19(32.2)$ & $40(67.8)$ & \\
\hline
\end{tabular}

a (Dermatology, dental, rheumatology, and neurology)

*Fisher's Exact test

\section{Phase C-Effect of tourniquet material on contamination} rate

All tourniquets (commercially available fabric tourniquets and plastic tubing) gave rise to bacterial growth on blood agar after single use. The mean CFU/ $\mathrm{cm}^{2}$ for all types of bacteria on plastic tubing was 3.68 (SD \pm 5.29; range 0.07-23.94) and for commercially available fabric tourniquets 2.25 ( $\mathrm{SD} \pm 4$.18; range $0.1-28.42$ ). The difference was not significant $(p=0.14)$. Two percent of plastic tubing and $6 \%$ of commercially available fabric tourniquets were contaminated with MRSA although the difference was not significant $(p=0.62$ ).

\section{Observation of standard precautions adopted during} phlebotomy

We made a total of 96 observations on health-care workers from 24 wards. During a single phlebotomy session, the maximum number of patients a single healthcare worker handled was 10 and in some wards it was one or two. Over $90 \%$ of health-care workers did not wash their hands before the procedure and $75 \%$ did not wash their hands after completing the phlebotomy procedure. Only one health-care worker washed hands both before and after handling the patient.

Thirty (68.8\%) health-care workers did not wear gloves during phlebotomy. Of those who did, none changed gloves between patients. A few (7/30) continued to wear gloves after phlebotomy. Of those who wore gloves, only one washed hands before and after wearing gloves and seven washed only after removing gloves.
Some health-care workers (19.8\%) continued to wear wristwatches or jewellery on their forearms or hands while performing phlebotomy.

Most of the health-care workers who washed their hands after the procedure used bar soap and all surfaces of the hands were lathered adequately. However, none of them rubbed hands systematically both before and after the procedure. One health-care worker used a disinfectant hand rub and the other rinsed only with water. All of them rinsed their hands adequately and most (16/19) dried their hands on towels or tissues that were available. Single use towels or tissues were provided in about half the units and health-care workers discarded them appropriately.

We observed that all plastic tubing were re-used on multiple patients without cleaning in between phlebotomy procedure. None of the tourniquets were visibly blood stained. When not in use, the tourniquets were often kept tied to the trolley that carried instruments and less frequently were placed on either trays/plastic containers, bench tops or patients' lockers or drawers or were hung on the walls.

\section{Discussion}

The rate of MRSA contamination of tourniquets in this hospital (25.7\%) was high. Other studies on re-usable tourniquets with sample size of $\geq 100$ have reported MRSA contamination rates ranging from $0 \%$ to $24 \%$ [2,3,6,14]. One study reported that the contamination of tourniquets which had been in use for ten days to two months was $24 \%$ [2]. However, in our study a contamination rate of 
$25.7 \%$ was found despite the tourniquets being used for not more than three days, indicating that the duration of use may not affect the contamination rate of tourniquets. This is further supported by our findings that single patient tourniquets had a similar rate of contamination (28.6\%) to that of reused tourniquets (25.5\%). The high MRSA contamination rate in single patient plastic tubing could be due to environmental contamination, as these are made from discarded infusion sets which have been lying in discard bins before being used as tourniquets. It has been previously suggested that contamination of tourniquets can be used as a surrogate marker of environmental contamination and the efficiency of infection control practices [6]. Our study also showed that re-used tourniquets were improperly stored and were likely to be contaminated from the environment.

There was no significant difference in contamination rates of re-used and single-patient tourniquets $(p=0.76)$. This has been reported previously. Pinto et al found that the highest rates of multi-resistant organisms were in single patient tourniquets in the intensive care unit. He concluded that it is not necessarily re-use that leads to contamination [6]. This concurs with our findings. Another study reported that tourniquets were contaminated mainly by the users' hands rather than the patients' skin and that MRSA contamination of tourniquets was significantly lower after hand hygiene education [2]. Poor hand hygiene of health-care workers could have contributed to the high rate of MRSA contamination of tourniquets in our study. The possibility that MRSA in the environment and fomites could lead to contamination of workers' hands should be a borne in mind [9]. Re-use of tourniquets enables cross contamination among patients and therefore, single use tourniquets are preferable. However, whether tourniquets actually increase the rate of hospital infection is ungertain $[$ [8] contradicting evidence regarding survival of MRSA on different types of surfaces. Some studies indicate that higher contamination rates and longer survival of MRSA occurs on rough or corrugated surfaces compared to smooth surfaces $[12,13]$. Other studies suggest that microscopic deep crevices on apparently smooth non-PVC plastic help sequester cells and protect them from dehydration [15]. In Phase C of our study (which showed no difference in the contamination rates between the two types of tourniquets) plastic tubing which was tied around patient's arm were handled more by the healthcare workers than the commercially available fabric tourniquets which required only pulling through a buckle to tighten. We believe that this increased handling and possible protection of bacteria by microscopic crevices on the apparently smooth surface of plastic tubing could have increased the contamination rate.

For a middle income country like Sri Lanka, commercially available tourniquets may not be a cost effective option. Therefore, we suggest using plastic tubing which is one seventh of the the cost of the former and replacing it after a single use. However, use of old infusion-set tubes is not advisable as these could already be contaminated. Single use alone is not sufficient to reduce cross-contamination, as a health-care worker with poor hand hygiene could transmit pathogens from the patient to a new tourniquet. Hand washing before and after handling a patient is the most effective means of preventing this cross-contamination. However, as in previous studies, our study also showed a low compliance by health-care workers regarding routine hand hygiene, which could be one reason for the high MRSA contamination rate in single use tourniquets $[16,17]$.

The contamination rate of faucets was higher in our study (26\%) than what has been previously reported (11\%) [4]. Contamination of skin or wounds via hands contaminated by faucets may lead to grave consequences, especially in the dermatological, surgical and gynaecology and obstetrics wards. As non-contact faucets are not a viable option for Sri Lanka, we suggest cleaning faucets regularly with detergent and encouraging health-care workers to close the taps using disposable tissues.

CHROMagar $^{\mathrm{TM}}$ MRSA medium (CHROMagar Microbiology, Paris, France) was used as a selective medium in this study as a large number of specimens had to be processed per day. It is more than $94.5 \%$ sensitive (24hrs incubation) and more than $96 \%$ specific as reported by several investigators who have evaluated this medium against standard methods $[18,19,20]$.

Considering the high contamination rate of tourniquets we recommend that a study should be carried out to determine if infection rates can be brought down by single use of tourniquets.

\section{Conclusion}

Our findings add to the existing evidence that reusable tourniquets and faucets act as reservoirs of MRSA. Single-use (i.e. disposed after single use) plastic tubing from new infusion sets were much less contaminated with MRSA than reused plastic tubing tourniquets and re-used tourniquets dedicated to a single patient. Universal standard hand-hygiene practices must be inbuilt in to the assessment of health-care workers in hospitals to minimise cross-contamination with MRSA.

\section{Funding}

The study was supported by University of Sri Jayewardenepura Research Grant (ASP/06/RE/MED/ 2012/29).

\section{Acknowledgements}

We thank the Director, consultants and nurses for their co-operation. We acknowledge assistance from the laboratory staff of the Department of Microbiology and funding received from the University of Sri Jayewardenepura (ASP/06/RE/MED/2012/29). 


\section{Conflicts of interest}

There are no conflicts of interest.

\section{References}

1. Haley RW, Hightower AW, Khabbaz RF, et al. The emergence of methicillin-resistant Staphylococcus aureus infections in United States hospitals. Possible role of the house staff-patient transfer circuit. Ann Intern Med 1982; 97: 297-308.

2. Leitch A, McCormick I, Gunn I, Gillespie T. Reducing the potential for phlebotomy tourniquets to act as a reservoir for methicillin-resistant Staphylococcus aureus. J Hosp Infect 2006; 63: 428-31.

3. Hensley DM, Krauland KJ, Mcglasson DI. Acinetobacter baumannii and MRSA contamination on reusable phlebotomy tourniquets. Clin Lab Sci 2010; 23: 151-6.

4. Bures S, Fishbain JT, Uyehara CFT, Parker JM, Berg BW. Computer keyboards and faucet handles as reservoirs of nosocomial pathogens in the intensive care unit. Am J Infect Control 2000; 28: 465-71.

5. Song JH, Hsue PR, Chung DR, Soo Ko K, Kang CI, Ran Peck K et al. Spread of methicillin-resistant Staphylococcus aureus between the community and the hospitals in Asian countries: an ANSORP study. J Antimicrob Chemother 2011; 66: 1061-69.

6. Pinto AN, Phan T, Sala G, Cheong EYL, Siarakas S, Gottlieb T. Reusable venesection tourniquets: a potential source of hospital transmission of multiresistant organisms. MJA 2011; 195: 276-9.

7. Elhassan HA, Dixon T. MRSA contaminated venepuncture tourniquets in clinical practice. Postgrad M J 2012; 88: 194-7.

8. Uneke CJ. Are non-critical medical devices potential sources of infections in healthcare facilities? World Health Popul 2014; 15(3): 13-24.

9. Bhalla A, Pultz NJ, Gries DM, Ray AJ, Eckstein EC, Aron DC, et al. Acquisition of nosocomial pathogens on hands after contact with environmental surfaces near hospitalised patients. Infect Control Hosp Epidemiol 2004; 25: 164-7.

10. Layton MC, Perez M, Heald P, Patterson JE. An outbreak of mupirocin-resistant Staphylococcus aureus on a dermatology ward associated with an environmental reservoir. Infect Control Hosp Epidemiol 1993; 14: 369-75.

11. Rampling A, Wiseman S, Davis L, Hyett AP, Walbridge AN, Payne GC, Cornaby AJ. Evidence that hospital hygiene is important in the control of methicillin-resistant Staphylococcus aureus. J Hosp Infect 2001; 49: 109-16.

12. Mehmood Z, Mubeen SM, Afzal MS, Hussain Z. Potential Risk of cross-infection by tourniquets: A Need for Effective Control Practices in Pakistan. Int J Prev Med 2014; 5: 1119-24.

13. Huang R, Mehta S, Weed D, Price CS. Methicillin resistant Staphylococcus aureus survival on hospital fomites. Infect Cont Hosp Epidemiol 2006; 27(11): 1267-9.

14. Rourke C, Bates C, Read RC. Poor hospital infection control practice in venepuncture and use of tourniquets. $J$ Hosp Infect 2001; 49: 59-61.

15. Coughenour C, Stevens V, Linda D, Stetzenbach LD. An evaluation of Methicillin-resistant Staphylococcus aureus survival on five environmental surfaces. Microb Drug Resist 2011; 17: 457-61.

16. World Health Organization. WHO guidelines on hand hygiene in health care (First global patient safety challenge clean care is safer care). WHO, 2009 http://apps.who.int/ iris/bitstream/10665/44102/1/9789241597906_eng.pdf (accessed on Sep 14, 2015).

17. Corea E, Ameen N, Rajakaruna S, Chandrasiri P. An audit on the technique of collection of blood for culture at the National Hospital of Sri Lanka. Sri Lankan Journal of Infectious Diseases 2011; 1: 18-23.

18. Diederen B, van Duijn I, van Belkum A, Willemse P, van Keulen P, Kluytmans J. Performance of CHROMagar MRSA Medium for Detection of Methicillin-Resistant Staphylococcus aureus. J Clin Microbiol 2005; 43: 1925-7.

19. Luteijn JM, Hubben GAA, Pechlivanoglou P, Bonten MJ, Postma MJ. Diagnostic accuracy of culture-based and PCRbased detection tests for methicillin-resistant Staphylococcus aureus: a meta-analysis. Clin Microbiol Infect 2011; 17: $146-54$.

20. Malhotra-Kumar S, Abrahantes JC, Sabiiti W, et al, Evaluation of Chromogenic Media for Detection of Methicillin-Resistant Staphylococcus aureus. J Clin Microbiol 2010; 48(4): 1040-6. 